\title{
TADPOLES AND SYMMETRIES IN HIGGS-GAUGE UNIFICATION THEORIES *
}

\author{
C. BIGGIO \\ Institut de Física d'Altes Energies (IFAE), \\ Universitat Autònoma de Barcelona, \\ E-08193 Bellaterra (Barcelona), SPAIN \\ E-mail: biggio@ifae.es \\ M. QUIRÓS \\ Institució Catalana de Recerca $i$ Estudis Avançats (ICREA) and \\ Institut de Física d'Altes Energies (IFAE), \\ Universitat Autònoma de Barcelona, \\ E-08193 Bellaterra (Barcelona), SPAIN \\ E-mail: quiros@ifae.es
}

\begin{abstract}
In theories with extra dimensions the Standard Model Higgs fields can be identified with internal components of bulk gauge fields (Higgs-gauge unification). The bulk gauge symmetry protects the Higgs mass from quadratic divergences, but at the fixed points localized tadpoles can be radiatively generated if $U(1)$ subgroups are conserved, making the Higgs mass UV sensitive. We show that a global symmetry, remnant of the internal rotation group after orbifold projection, can prevent the generation of such tadpoles. In particular we consider the classes of orbifold compactifications $T^{d} / \mathbb{Z}_{N}(d$ even, $N>2)$ and $T^{d} / \mathbb{Z}_{2}$ (arbitrary $\left.d\right)$ and show that in the first case tadpoles are always allowed, while in the second they can appear only for $d=2$ (six dimensions).
\end{abstract}

\section{Introduction}

Among the possible motivations for studying theories in extra dimensions with Higgs-gauge unification ${ }^{1-4}$ there is the so called little hierarchy problem $^{5}$. The latter consists in the one order of magnitude discrepancy between the upper bound for the Standard Model cutoff $\Lambda_{E W}$ coming from

*Based on talks given by M.Q. at String Phenomenology 2004, University of Michigan, Ann Arbor, August 1-6, 2004 and 10th International Symposium on Particles, Strings and Cosmology (PASCOS'04 and Nath Fest), Northeastern University, Boston, August 16-22, 2004. 
the requirement of stability of the Higgs mass under radiative corrections and the lower bound arising from the non-observation of dimension-six fourfermion operators ${ }^{6}$.

Up to now the best solution to the little (and grand) hierarchy problem is supersymmetry. Indeed in this framework the Standard Model cutoff is identified with the mass of supersymmetric particles, while $R$-parity conservation induces a suppression in the loop corrections to four-fermion operators which solves the little hierarchy problem. However, since the minimal supersymmetric Standard Model extension is becoming very constrained, it is useful to propose possible alternative solutions which may fill the gap between the sub- $\mathrm{TeV}$ scale required for the stability of the electroweak symmetry breaking and the multi- $\mathrm{TeV}$ scale required by precision tests of the Standard Model.

One possible alternative solution is Higgs-gauge unification. In these theories the internal components of higher dimensional gauge bosons play the role of the Standard Model Higgses and can acquire a non-vanishing vacuum expectation value through the Hosotani mechanism ${ }^{7}$. The Higgs mass in the bulk is protected from quadratic divergences by the higherdimensional gauge theory and only finite corrections $\propto(1 / R)^{2}(R$ is the compactification radius) can appear. The Standard Model cutoff is then identified with $1 / R$ and the little hierarchy between $1 / R$ and $\Lambda$, which is now the cutoff of the higher dimensional theory, is protected by the higherdimensional gauge symmetry. However at the fixed points the bulk gauge symmetry is broken and localized terms consistent with the residual symmetries can be generated by quantum corrections ${ }^{8}$. While a direct localized squared mass $\left(\sim \Lambda^{2}\right)$ for the Higgs-gauge fields is forbidden by a shift symmetry remnant of the original bulk gauge symmetry ${ }^{2}$, if a $U(1)$ symmetry is conserved at a given fixed point then the corresponding field strength can be radiatively generated, giving rise to a quadratic divergent mass for the Higgs ${ }^{2,3}$. This is a generic feature of orbifold compactifications in dimensions $D \geq 6$ and has been confirmed in six-dimensional orbifold field ${ }^{4}$ and ten-dimensional string ${ }^{9}$ theories. One way out ${ }^{4}$ is that local tadpoles vanish globally, but a more elegant way, explored in Ref. [10] and discussed in this talk, is to find a symmetry which forbids the generation of these localized terms. This symmetry is precisely the subgroup of the tangent space group $S O(D-4)$ whose generators commute with the orbifold group elements leaving the considered fixed point invariant. How this symmetry comes out and how it is related to the generation of localized tadpoles will be discussed in the following. 


\section{Symmetries at the fixed points and allowed localized terms}

We consider a gauge theory (gauge group $\mathcal{G}$ ) coupled to fermions in a $D=$ $d+4>4$ dimensional space-time parametrized by coordinates $x^{M}=\left(x^{\mu}, y^{i}\right)$ where $\mu=0,1,2,3$ and $i=1, \ldots, d$. The Lagrangian is

$$
\mathcal{L}_{D}=-\frac{1}{4} F_{M N}^{A} F^{A M N}+i \bar{\Psi} \Gamma_{D}^{M} D_{M} \Psi,
$$

with $F_{M N}^{A}=\partial_{M} A_{N}^{A}-\partial_{N} A_{M}^{A}-g f^{A B C} A_{M}^{B} A_{N}^{C}, D_{M}=\partial_{M}-i g A_{M}^{A} T^{A}$ and where $\Gamma_{D}^{M}$ are the $\Gamma$-matrices corresponding to a $D$-dimensional space-time. The local symmetry of (1) is the invariance under the (infinitesimal) gauge transformations

$$
\delta_{\xi} A_{M}^{A}=\frac{1}{g} \partial_{M} \xi^{A}-f^{A B C} \xi^{B} A_{M}^{C}, \quad \delta_{\xi} \Psi=i \xi^{A} T^{A} \Psi .
$$

Now we compactify the extra dimensions on an orbifold. Firstly we construct a $d$-dimensional torus $T^{d}$ by modding out $\mathbb{R}^{d}$ by a $d$-dimensional lattice $\Lambda^{d}$ and then we define the orbifold by modding out $T^{d}$ by $\mathbb{G}$, where $\mathbb{G}$ is a discrete symmetry group acting non-freely (i.e. with fixed points) on it ${ }^{11}$. The orbifold group is generated by a discrete subgroup of $S O(d)$ that acts crystallographically on the torus lattice and by discrete shifts that belong to the torus lattice. The action of $k \in \mathbb{G}$ on the torus is $k \cdot y=P_{k} y+u$, where $P_{k}$ is a discrete rotation in $S O(d)$ and $u \in \Lambda^{d} ; y$ and $k \cdot y$ are then identified on the orbifold. Since the orbifold group is acting non-freely on the torus there are fixed points characterized by $k \cdot y_{f}=y_{f}$. Any given fixed point $y_{f}$ remains invariant under the action of a subgroup $\mathbb{G}_{f}$ of the orbifold group.

The orbifold group acts on fields $\phi_{\mathcal{R}}$ transforming as an irreducible representation $\mathcal{R}$ of the gauge group $\mathcal{G}$ as

$$
k \cdot \phi_{\mathcal{R}}(y)=\lambda_{\mathcal{R}}^{k} \otimes \mathcal{P}_{\sigma}^{k} \phi_{\mathcal{R}}\left(k^{-1} \cdot y\right)
$$

where $\lambda_{\mathcal{R}}^{k}$ is acting on gauge and flavor indices and $\mathcal{P}_{\sigma}^{k}$, where $\sigma$ refers to the field spin, on Lorentz indices. In particular one finds for scalar fields $\mathcal{P}_{0}^{k}=1$ and for gauge fields $\mathcal{P}_{1}^{k}=P_{k}$ for a discrete rotation $\left(\mathcal{P}_{1}^{k}=1\right.$ for a lattice shift), while for fermions $\mathcal{P}_{\frac{1}{2}}^{k}$ can be derived requiring the invariance of the lagrangian under the orbifold action. On the other side $\lambda_{\mathcal{R}}^{k}$ depends on the gauge structure and the gauge breaking of the orbifold action.

In general the orbifold action breaks the gauge group in the bulk $\mathcal{G}=$ $\left\{T^{A}\right\}$ to a subgroup $\mathcal{H}_{f}=\left\{T^{a_{f}}\right\}$ at the fixed point $y_{f}$. It can be shown that the subgroup $\mathcal{H}_{f}$ left invariant by the orbifold elements $k \in \mathbb{G}_{f}$ is 
defined by the generators that commute with $\lambda_{\mathcal{R}}^{k}$, i.e. $\left[\lambda_{\mathcal{R}}^{k}, T_{\mathcal{R}}^{a_{f}}\right]=0$. The latter condition must be satisfied by any irreducible representation $\mathcal{R}$ of $\mathcal{G}$.

We now consider the effective four-dimensional lagrangian. This can be written as:

$$
\mathcal{L}_{4}^{e f f}=\int d^{d} y\left[\mathcal{L}_{D}+\sum_{f} \delta^{(d)}\left(y-y_{f}\right) \mathcal{L}_{f}\right]
$$

where $\mathcal{L}_{D}$ is given by (1) and $\mathcal{L}_{f}$ is the most general lagrangian consistent with the symmetries localized at the fixed point $y_{f}$. In order to write the most general $\mathcal{L}_{f}$ we need to know which are the symmetries present at each fixed point. First of all the operators must be invariant under the action of the orbifold group $\left[\mathbb{G}_{f}\right]$ and the $4 \mathrm{D}$ Lorentz group $[S O(1,3)]$. Then we have to consider the bulk gauge symmetry $\mathcal{G}$ : when applied to the orbifold fixed points $y_{f}$ it reduces to the four-dimensional gauge symmetry $\mathcal{H}_{f}=\left\{T^{a_{f}}\right\}$ that applies to the four-dimensional gauge fields $A_{\mu}^{a_{f}}$ which are also invariant under the orbifold action. This consists in the usual gauge invariance under $\mathcal{H}$-transformations ${ }^{\mathrm{a}} \delta_{\xi} A_{\mu}^{a}=\partial_{\mu} \xi^{a} / g-f^{a b c} \xi^{b} A_{\mu}^{c}$. However this is not the only symmetry generated by the original gauge symmetry $\mathcal{G}$. Indeed by localizing the transformations (2) at the orbifold fixed point $y_{f}$ and keeping the orbifold invariant terms one can define an infinite set of transformations (remnant of the bulk gauge invariance) induced by derivatives of $\xi^{A}$ that we can call $\mathcal{K}$-transformations ${ }^{2}$. Then only $\mathcal{H}$ and $\mathcal{K}$-invariant quantities are allowed at the orbifold fixed points.

The presence of the remnant gauge symmetry $\mathcal{K}$ is very important in order to prevent the appearance of direct mass terms for gauge fields localized at the orbifold fixed points. Indeed if the gauge field $A_{i}^{\hat{a}}$ is invariant under the orbifold action, where $T^{\hat{a}} \in \mathcal{G} / \mathcal{H}$, the remnant "shift" symme$\operatorname{try} \delta_{\xi} A_{i}^{\hat{a}}=\partial_{i} \xi^{\hat{a}} / g-f^{\hat{a} b \hat{c}} \xi^{b} A_{i}^{\hat{c}}$ prevents the corresponding zero mode from acquiring a localized mass.

Now that we know which are the symmetries at the fixed points we can write the most general lagrangian $\mathcal{L}_{f}$. Invariant operators are $\left(F_{\mu \nu}^{a}\right)^{2}$, which corresponds to a localized kinetic term for $A_{\mu}^{a}$ and $F_{\mu \nu}^{a} \tilde{F}_{\mu \nu}^{a}$ which is a localized anomaly. Moreover if for some $(i, j) F_{i j}^{a}$ is orbifold invariant (this is model-dependent), $\left(F_{i j}^{a}\right)^{2}$ can be non-zero at a fixed point and if also $A_{i}^{\hat{a}}$ is orbifold invariant, $\left(F_{i \mu}^{\hat{a}}\right)^{2}$ can be present too. These last two lagrangian terms give rise, respectively, to localized quartic couplings and localized

aFrom here on we will remove for simplicity the subscript "f" from the gauge group and the corresponding generators. 
kinetic terms for $A_{i}^{\hat{a}}$. All these operators are dimension four, that is they renormalize logarithmically. However if $\mathcal{H}$ contains a $U(1)$ factor

$$
F_{i j}^{\alpha}=\partial_{i} A_{j}^{\alpha}-\partial_{j} A_{i}^{\alpha}-g f^{\alpha \hat{b} \hat{c}} A_{i}^{\hat{b}} A_{j}^{\hat{c}},
$$

where $\alpha$ is the $U(1)$ quantum number, is $\mathcal{H}$ and $\mathcal{K}$-invariant as well as orbifold and Lorentz invariant. If this operator is allowed, we expect that both a tadpole for the derivative of odd fields, $\partial_{i} A_{j}^{a}$, and a mass term for the even fields, $f^{a \hat{b} \hat{c}} A_{i}^{\hat{b}} A_{j}^{\hat{c}}$, will be generated on the brane by bulk radiative corrections. Moreover, since these operators have dimension two, we expect that their respective renormalizations will lead to quadratic divergences, making the theory ultraviolet sensitive.

Apart from the case of $D=5$ where the term $F_{i j}$ does not exist, for $D \geq 6$ it does and we expect the corresponding mass terms to be generated on the brane by radiative corrections. This has been confirmed by direct computation in six-dimensional orbifold field ${ }^{2-4}$ and ten-dimensional string $^{9}$ theories. Of course if these divergent localized mass terms were always present, Higgs-gauge unification theories would not be useful in order to solve the little hierarchy problem. One way out can be that local tadpoles vanish globally, but this requires a strong restriction on the bulk fermion content $^{4}$. A more elegant and efficient solution would be finding another symmetry forbidding the generation of localized tadpoles: this symmetry exists and has been studied in Ref. [10].

When compactifing a $d$-dimensional space to a smooth Riemannian manifold (with positive signature), a tangent space can be defined at each point and the orthogonal transformations acting on it form the group $S O(d)^{12}$. When an orbifold group acts on the manifold it also breaks the internal rotation group $S O(d)$ into a subgroup $\mathcal{O}_{f}$ at the orbifold fixed point $y_{f}$. Indeed here a further compatibility condition between the orbifold action and the internal rotations is required. In particular, if the given fixed point $y_{f}$ is left invariant by the orbifold subgroup $\mathbb{G}_{f}$, only $\mathbb{G}_{f}$-invariant operators $\Phi_{\mathcal{R}, \sigma}$ couple to $y_{f}$, i.e.

$$
k \cdot \Phi_{\mathcal{R}, \sigma}\left(y_{f}\right)=\Phi_{\mathcal{R}, \sigma}\left(y_{f}\right) .
$$

Acting on $\Phi_{\mathcal{R}, \sigma}$ with an internal rotation we get a transformed operator that should also be $\mathbb{G}_{f}$-invariant. This means, using Eq. (3), that the subgroup $\mathcal{O}_{f}$ is spanned by the generators of $S O(d)$ that commute with $\mathcal{P}_{\sigma}^{k}$, i.e. they satisfy the condition

$$
\left[\mathcal{O}_{f}, \mathcal{P}_{\sigma}^{k}\right]=0
$$


for $k \in \mathbb{G}_{f}$ and arbitrary values of $\sigma$. In particular in the presence of gauge fields $A_{M}=\left(A_{\mu}, A_{i}\right)$ an invariant operator can be $F_{i j}$ with $\mathcal{R}=A d j$ and $\sigma=2$. The internal components $A_{i}$ transform under the action of the orbifold element $k \in \mathbb{G}_{f}$ as the discrete rotation $P_{k}$. At the orbifold fixed point $y_{f}$ only the subgroup $\mathcal{O}_{f} \subseteq S O(d)$ survives and the vector representation $A_{i}$ of $S O(d)$ breaks into irreducible representations of $\mathcal{O}_{f}$.

We have then identified an additional symmetry that the lagrangian $\mathcal{L}_{f}$ at the fixed point $y_{f}$ must conserve. Summarizing, the invariances that we have to take into account are the following: four-dimensional Lorentz invariance $[S O(1,3)]$, invariance under the action of the orbifold group $[\mathbb{G}]$, usual four-dimensional gauge invariance $[\mathcal{H}]$, remnant of the bulk gauge invariance $[\mathcal{K}]$ and invariance under rotations of the tangent space $\left[\mathcal{O}_{f}\right]$.

Now we consider the tadpole term of Eq. (5) in order to see if and when it is invariant under the last discussed symmetry. We have just seen that the vector representation $A_{i}$ of $S O(d)$ breaks into irreducible representations of the internal rotation group $\mathcal{O}_{f} \subseteq S O(d)$. In particular if the rotation subgroup acting on the $(i, j)$-indices is $S O(2)$ then $\epsilon^{i j} F_{i j}^{\alpha}$, where $\epsilon^{i j}$ is the Levi-Civita tensor, is invariant under $\mathcal{O}_{f}$ and so it can be radiatively generated. On the other hand if the rotation subgroup acting on the $(i, j)$-indices is $S O(p)(p>2)$ then the Levi-Civita tensor would be $\epsilon^{i_{1} i_{2} \ldots i_{p}}$ and only invariants constructed using p-forms would be allowed. In other words a sufficient condition for the absence of localized tadpoles is that the smallest internal subgroup factor be $S O(p)(p>2)$.

\section{Tadpoles for $T^{D} / \mathbb{Z}_{N}$ orbifolds}

To show explicitly how the above discussed symmetry arguments apply, we consider the class of orbifolds $\mathbb{G}=\mathbb{Z}_{N}$ for even $d$. The generator $P_{N}$ of the orbifold group is defined by

$$
P_{N}=\prod_{i=1}^{d / 2} e^{2 \pi i \frac{k_{i}}{N} J_{2 i-1,2 i}}
$$

where $k_{i}$ are integer numbers $\left(0<k_{i}<N\right)$ and $J_{2 i-1,2 i}$ is the generator of a rotation with angle $2 \pi \frac{k_{i}}{N}$ in the plane $\left(y_{2 i-1}, y_{2 i}\right)$. All orbifold elements are defined by $P_{k}=P_{N}^{k}(k=1, \ldots, N-1)$ and satisfy the condition $P_{N}^{N}=1$. The generator of rotations in the $\left(y_{2 i-1}, y_{2 i}\right)$-plane can be written as $J_{2 i-1,2 i}=\operatorname{diag}\left(0, \ldots, \sigma^{2}, \ldots, 0\right)$ where the Pauli matrix $\sigma^{2}$ is in the $i$-th two-by-two block. Therefore the generator $P_{N}$ can be written as $P_{N}=$ $\operatorname{diag}\left(R_{1}, \ldots, R_{d / 2}\right)$ where the discrete rotation in the $\left(y_{2 i-1}, y_{2 i}\right)$-plane is 
defined as

$$
R_{i}=\left(\begin{array}{cc}
c_{i} & s_{i} \\
-s_{i} & c_{i}
\end{array}\right)
$$

with $c_{i}=\cos \left(2 \pi k_{i} / N\right), s_{i}=\sin \left(2 \pi k_{i} / N\right)$.

Let $y_{f}$ be a fixed point that is left invariant under the orbifold subgroup $\mathbb{G}_{f}=\mathbb{Z}_{N_{f}}$ where $N_{f} \leq N$. We now define the internal rotation group $\mathcal{O}_{f}$ as the subgroup of $S O(d)$ that commutes with the generator of the orbifold $\mathbb{Z}_{N_{f}}, P_{N_{f}}$ as given by Eq. (8) with $N$ replaced by $N_{f}$. In general, if $N_{f}>2$ $\mathcal{O}_{f}$ is trivially provided by the tensor product:

$$
\mathcal{O}_{f}=\bigotimes_{i=1}^{d / 2} S O(2)_{i}
$$

where $S O(2)_{i}$ is the $S O(2) \subseteq S O(d)$ that acts on the $\left(y_{2 i-1}, y_{2 i}\right)$-subspace. In every such subspace the metric is $\delta_{I J}$ and the Levi-Civita (antisymmetric) tensor $\epsilon^{I J}(I, J=2 i-1,2 i, i=1, \ldots, d / 2)$ such that we expect the tadpoles appearance at the fixed points $y_{f}$ as

$$
\sum_{i=1}^{d / 2} \mathcal{C}_{i} \sum_{I, J=2 i-1}^{2 i} \epsilon^{I J} F_{I J}^{\alpha} \delta^{(d / 2)}\left(y-y_{f}\right) .
$$

If $N_{f}=2$ then the generator of the orbifold subgroup $\mathbb{G}_{f}=\mathbb{Z}_{2}$ is the inversion $P=-\mathbf{1}$ that obviously commutes with all generators of $S O(d)$ and $\mathcal{O}_{f}=S O(d)$. In this case the Levi-Civita tensor is $\epsilon^{i_{1} \ldots i_{d}}$ and only a d-form can be generated linearly in the localized lagrangian. Therefore tadpoles are only expected in the case of $d=2(D=6)$.

The last comments also apply to the case of $\mathbb{Z}_{2}$ orbifolds of arbitrary dimensions (even or odd) since in that case the orbifold generator is always $P=-\mathbf{1}$ and the internal rotation group that commutes with $P$ is $\mathcal{O}_{f}=$ $S O(d)$ for all the fixed points. Again tadpoles are only expected for $D=6$ dimensions while they should not appear for $D>6$.

Since every operator in $\mathcal{L}_{f}$ that is consistent with all the symmetries should be radiatively generated by loop effects from matter in the bulk (unless it is protected by some other - accidental- symmetry) explicit calculations of the tadpole in orbifold gauge theories should confirm the appearance (or absence) of them in agreement with the above symmetry arguments.

In Ref. [10] we have considered the class of orbifold compactifications $T^{d} / \mathbb{Z}_{2}$ and we have explicitly calculated the tadpole at one- and two-loop level. The result we found, in agreement with our general conclusions, 
is that the tadpole is zero for $D>6$, while it can be non-zero only for $D=6$. In particular we found that gauge and ghost contributions are always zero, even in six dimensions, due to an extra parity symmetry that inverts separately the internal coordinates. As for the fermion contribution, it is always zero except for $D=6$, where it is proportional to $\epsilon^{i j}$, in agreement with Eq. (11). Since in six dimensions there are two possibilities for $\mathcal{P}_{\frac{1}{2}}$, we observed that in one case the result is chiral-independent, while in the other the sign depends on the six dimensional chirality of fermions. In the latter case this means that starting with Dirac fermions can imply a vanishing tadpole. Unfortunately, due to the existing relation between the tadpole and the mixed $U(1)$-gravitational anomaly, a vanishing tadpole corresponds to a non-vanishing anomaly. The condition for a vanishing tadpole coincides with the one for a vanishing anomaly only when dealing with chiral fermions of equal six-dimensional chirality, where this reduces to

$$
\operatorname{tr}\left\{\lambda_{\mathcal{R}} T_{\mathcal{R}}^{A}\right\}=0
$$

\section{Conclusions and outlook}

In orbifold field theories Standard Model Higgs fields can be identified with the internal components $A_{i}$ of bulk gauge fields. Higher-dimensional gauge invariance prevents the Higgs from acquiring a quadratically divergent mass in the bulk while a shift symmetry, remnant of the bulk gauge symmetry at the fixed points, forbids it on the branes. Nevertheless, if a $U(1)$ subgroup is conserved at the fixed points, the corresponding field strength is gauge invariant and can be radiatively generated giving rise, through its non-abelian part, to a quadratically divergent mass for the Higgs. However an additional symmetry must be taken into account. Indeed, being the Higgs fields identified with the internal components of bulk gauge fields, they transform in the vector representation of the tangent space rotation group $S O(d)$. This is valid in general on a $d$-dimensional compact manifold, but at the orbifold fixed points the orbifold projection must be taken into account. This induces a reduction of the tangent space group to the subgroup $\mathcal{O}_{f}$ whose generators commute with the orbifold subgroup leaving the corresponding fixed points invariant. If $\mathcal{O}_{f}=S O(2)$, then the Levi-Civita tensor with two indices $\epsilon^{i j}$ exists and the corresponding field strength can be generated on the brane as $\epsilon^{i j} F_{i j}$. On the contrary if $\mathcal{O}_{f} \supseteq S O(p)$ with $p>2$ then only invariant terms built with the $p$-form $\epsilon^{i_{1} \ldots i_{p}}$ are allowed and so the tadpole cannot be generated. We have shown that in the class 
of orbifolds $T^{d} / \mathbb{Z}_{N}(d$ even, $N>2) \mathcal{O}_{f}=S O(2) \otimes S O(2) \otimes \cdots \otimes S O(2)$, i.e. tadpoles are always allowed. On the contrary on orbifolds $T^{d} / \mathbb{Z}_{2}$ (arbitrary $d) \mathcal{O}_{f}=S O(d)$ that means that tadpoles can be generated only in six dimensions $(d=2)$.

However the absence of tadpoles seems to be a necessary but not sufficient condition in order to build a realistic theory of electroweak symmetry breaking without supersymmetry. First of all it seems compulsory considering models with more than six dimensions, since in five dimensions the absence of quartic coupling leads to too low Higgs masses, while in six dimensions electroweak symmetry breaking is spoiled by localized tadpoles. We have seen that for $T^{d} / \mathbb{Z}_{2}$ orbifolds with $d>2$ tadpoles cannot be generated, but in general these models predict the existence of $d$ Higgs fields, leading to non-minimal models. Of course the conditions that preclude the existence of quadratic divergences for Higgs fields do not forbid the radiative generation of finite $\sim(1 / R)^{2}$ masses, that vanish in the $R \rightarrow \infty$ limit. Some of the above Higgs fields can acquire different masses and even not participate in the electroweak symmetry breaking phenomenon, depending on the models. Moreover, even if we were able to build a model with only one Standard Model Higgs field, its mass should be in agreement with LEP bounds and precision measurements.

Another issue that must be addressed in order to construct a realistic model is the flavour problem. One possibility should be putting matter fermions in the bulk and coupling them to an odd mass that localizes them at different locations ${ }^{13}$. This seems nice since, for instance, it can also be used to explain fermion replica ${ }^{14}$, but problems can arise with $\mathrm{CP}$ violation and flavour changing neutral currents ${ }^{15}$. Another possibility is to consider localized matter fermions which can develop Yukawa couplings through Wilson line interactions after the heavy bulk fermions have been integrated out $^{3,16}$

\section{References}

1. S. Randjbar-Daemi, A. Salam and J. Strathdee, Nucl. Phys. B 214 (1983) 491; I. Antoniadis and K. Benakli, Phys. Lett. B 326 (1994) 69 [arXiv:hepth/9310151]; H. Hatanaka, T. Inami and C. S. Lim, Mod. Phys. Lett. A 13 (1998) 2601 [arXiv:hep-th/9805067]; H. Hatanaka, Prog. Theor. Phys. 102 (1999) 407 [arXiv:hep-th/9905100]; M. Kubo, C. S. Lim and H. Yamashita, arXiv:hep-ph/0111327; G. R. Dvali, S. Randjbar-Daemi and R. Tabbash, Phys. Rev. D 65 (2002) 064021 [arXiv:hep-ph/0102307]; I. Antoniadis, K. Benakli and M. Quiros, Nucl. Phys. B 583 (2000) 35 [arXiv:hep-ph/0004091]; 
I. Antoniadis, K. Benakli and M. Quiros, New Jour. Phys. 3 (2001) 20 [arXiv:hep-th/0108005].

2. G. von Gersdorff, N. Irges and M. Quiros, Phys. Lett. B 551 (2003) 351 [arXiv:hep-ph/0210134].

3. C. Csaki, C. Grojean and H. Murayama, Phys. Rev. D 67 (2003) 085012 [arXiv:hep-ph/0210133].

4. C. A. Scrucca, M. Serone, L. Silvestrini and A. Wulzer, JHEP 0402 (2004) 049 [arXiv:hep-th/0312267]; A. Wulzer, arXiv:hep-th/0405168.

5. R. Barbieri and A. Strumia, arXiv:hep-ph/0007265; G. F. Giudice, Int. J. Mod. Phys. A 19 (2004) 835 [arXiv:hep-ph/0311344].

6. S. Eidelman et al., "Review of Particle Physics", Phys. Lett. B 592 (2004) 1.

7. Y. Hosotani, Phys. Lett. B 126 (1983) 309; Y. Hosotani, Annals Phys. 190 (1989) 233; N. Haba, Y. Hosotani and Y. Kawamura, Prog. Theor. Phys. 111 (2004) 265 [arXiv:hep-ph/0309088]; N. Haba, M. Harada, Y. Hosotani and Y. Kawamura, Nucl. Phys. B 657 (2003) 169 [Erratum-ibid. B 669 (2003) 381] [arXiv:hep-ph/0212035]; Y. Hosotani, S. Noda and K. Takenaga, Phys. Rev. D 69 (2004) 125014 [arXiv:hep-ph/0403106]; Y. Hosotani, S. Noda and K. Takenaga, arXiv:hep-ph/0410193.

8. H. Georgi, A. K. Grant and G. Hailu, Phys. Lett. B 506 (2001) 207 [arXiv:hepph/0012379]; H. Georgi, A. K. Grant and G. Hailu, Phys. Rev. D 63 (2001) 064027 [arXiv:hep-ph/0007350]; R. Contino, L. Pilo, R. Rattazzi and E. Trincherini, Nucl. Phys. B 622 (2002) 227 [arXiv:hep-ph/0108102]; G. von Gersdorff, N. Irges and M. Quiros, Nucl. Phys. B 635 (2002) 127 [arXiv:hepth/0204223].

9. S. Groot Nibbelink, H. P. Nilles, M. Olechowski and M. G. A. Walter, Nucl. Phys. B 665 (2003) 236 [arXiv:hep-th/0303101]; S. Groot Nibbelink and M. Laidlaw, JHEP 0401, 004 (2004) [arXiv:hep-th/0311013]; S. Groot Nibbelink and M. Laidlaw, JHEP 0401 (2004) 036 [arXiv:hep-th/0311015].

10. C. Biggio and M. Quiros, to appear in Nucl. Phys. B [arXiv:hep-ph/0407348].

11. L. J. Dixon, J. A. Harvey, C. Vafa and E. Witten, Nucl. Phys. B 261 (1985) 678; L. J. Dixon, J. A. Harvey, C. Vafa and E. Witten, Nucl. Phys. B 274 (1986) 285.

12. See e.g. M. B. Green, J. H. Schwarz and E. Witten, "Superstring Theory. Vol. 2: Loop Amplitudes, Anomalies And Phenomenology," Cambridge, Uk: Univ. Pr (1987) (Cambridge Monographs On Mathematical Physics).

13. G. Burdman and Y. Nomura, Nucl. Phys. B 656 (2003) 3 [arXiv:hep$\mathrm{ph} / 0210257]$.

14. C. Biggio, F. Feruglio, I. Masina and M. Perez-Victoria, Nucl. Phys. B 677 (2004) 451 [arXiv:hep-ph/0305129].

15. A. Delgado, A. Pomarol and M. Quiros, JHEP 0001 (2000) 030 [arXiv:hep$\mathrm{ph} / 9911252]$.

16. C. A. Scrucca, M. Serone and L. Silvestrini, Nucl. Phys. B 669 (2003) 128 [arXiv:hep-ph/0304220]. 